\author{
Rahim Mohammadi, Hamid Shariati, \\ Neda Sharghi and Maryam Naghipour \\ Department of Surgery and Diagnostic Imaging, \\ Faculty of Veterinary Medicine, Urmia University, \\ Nazloo Road, Urmia, 57153 1177, Iran \\ Dates: Received: 02 March, 2015; Accepted: 14 \\ May, 2015; Published: 16 May, 2015 \\ *Corresponding author: Rahim Mohammadi, \\ Department of Surgery and Diagnostic Imaging, \\ Faculty of Veterinary Medicine, Urmia University, \\ Nazloo Road, Urmia,57153 1177, Iran, Tel: +98 \\ 443 2770508; Fax: +98 443277 1926; E-mail: \\ r.mohammadi@urmia.ac.ir \\ www.peertechz.com
}

ISSN: 2455-4634

Keywords: Peripheral nerve repair; Sciatic Cyclosporin A; Local diabetes

\section{Research Article \\ Peripheral Nerve Regeneration using Silicone Rubber Chamber Combined by Local Administration of Cyclosporin a in Streptozotocin Induced Diabetic Rats}

\section{Abbreviations}

SIL: Silicon; Cs A: Cyclosporin A

\section{Introduction}

Surgical therapy in patients with peripheral nerve injuries has not presented changes over the last decades due to the use of autologous grafts, development of intraoperative magnification, and proven deleterious effects of tension at neural repair site and despite all the advancements achieved, functional repair results are still imperfect [1]. In case of significant damage to nerve tissue, severely-damaged nerves do not spontaneously restore their function and their continuity has to be first reestablished by microsurgical intervention such as suture or interposition of a graft [2,3]. A widely accepted method used by most surgeons involves bridging the defect with an autologous donor nerve. However, it has disadvantages of sacrificing functional nerves [4]. In extensive defects or in several nerve defects on a same patient, there may not be enough autologous donor nerve to fill that neural failure [1]. Therefore, increasing efforts have been made over the last two decades to investigate effective alternatives to autogenous nerve grafts [5]. The possibility of repairing nerve defects through bridging a gap by means of tubes made by nonnervous materials has been widely experienced, both experimentally and in clinical practice [5-7]. Many types of cells, growth factors and drugs have been used combined with tube grafting to promote nerve regeneration $[2,8]$.

It has been reported that using silicone tubes in bridging of nerve defects could be promising because it is inert and does not induce extensive scarring or degeneration after implantation [9]. The advantages like no donor morbidity, availability, affordability and no foreign reactions make silicone rubber chamber an attractive alternative to other standard grafts [10]. It has been demonstrated that silicone rubber tubes are well tolerated in humans even after 3 years of implantation [11].

Immunosuppressants were originally introduced to prevent rejection of allografts; later, it was discovered that FK506 also possessed nerve regenerative properties when applied systemically and topically loaded at sub-immunosuppressant doses in non-nerve grafts [12-15]. Cyclosporine has been widely employed in organ transplantations in association with other drugs, allowing a significant morbidity reduction when compared to early immunosuppression methods [16]. Mechanism of action of cyclosporine in nerve regeneration remains controversial [17-20]. The role of cyclosporine A (CsA) on peripheral nerve regeneration has been investigated after peripheral nerve allografting in experimental models immunosuppressed with CsA $[21,22]$. Most of these studies were concentrated on allograft 
survival, rather than on the direct effect of CsA on peripheral nerve regeneration [18]. The literature is poor and to the best of our knowledge local effect of CsA on peripheral nerve repair has not been well investigated to date on diabetic rats.

Because of promising beneficial effects of silicone tube as an inert conduit and the neuroregenerative and immunosuppressive effects of CsA, the objective of the present study was to assess topical effects of a CsA -loaded silicone tube as an in situ delivery system of CsA in bridging the defects on peripheral nerve regeneration using a rat sciatic nerve transection model. Assessment of nerve regeneration was based on behavioral, functional (walking track analysis), electrophysiological, histomorphometrical and immunohistochemical (Schwann cell detection by S-100 expression) assessments at $4,8,12$ and 16 weeks surgery.

\section{Materials and Methods}

\section{Experimental design}

Eighty male White Wistar rats weighing approximately $290 \mathrm{~g}$ were divided into four experimental groups $(\mathrm{n}=20)$, randomly: Transected control group (TC), sham-surgery group (Sham), silicone control group (SIL) and cyclosporin treated group (SIL/CsA). Each group was again subdivided into four subgroups of five animals each and surveyed in four time points of. Two weeks before and during the entire experiments, the animals were housed in individual plastic cages with an ambient temperature of $23 \pm 3^{\circ} \mathrm{C}$, stable air humidity, and a natural day/night cycle. The rats had free access to standard rodent laboratory food and tap water. For insulin-deficient diabetes, rats were fasted overnight before receiving a single intraperitoneal injection (50 mg/kg in $0.9 \%$ sterile saline) of streptozotocin (STZ). Hyperglycemia (15 mmol/l or greater) was confirmed 2 days later by measurement of tail-vein blood glucose concentration (Ames Glucostix; Myles, Elkhart, IN). The rats underwent grafting procedures three days after induction of diabetes.

\section{Preparation of cyclosporine A}

The dose of CsA was modification of a method described by others for other immunosuppressant [15]. Briefly, the original solution of CsA (Sigam-aldrich, Chemie GmbH and Germany) was prepared as $5 \mathrm{mg} / \mathrm{ml}$. Then two serial dilutions were performed to reduce the concentration to $10 \mathrm{ng} / \mathrm{ml}$ : carrier dilution $(20 \mu \mathrm{l}$ original CsA into 10 $\mathrm{ml}$ sterile olive oil [Sigma-aldrich Chemie GmbH, Germany] to give final concentration of $10 \mu \mathrm{g} / \mathrm{ml})$ and carrier-drug dilution $(10 \mu \mathrm{l}$ of first dilution into $10 \mathrm{ml}$ olive oil to give final concentration of $10 \mathrm{ng} /$ $\mathrm{ml}$ ). The natural viscosity of the oil maintained the carrier or carrierdrug combination within the silicon conduit.

\section{Grafting procedure}

Animals were anesthetized by intraperitoneal administration of ketamine-xylazine (ketamine hydrochloride $5 \%, 90 \mathrm{mg} / \mathrm{kg}$ and xylazine hydrochloride $2 \%, 5 \mathrm{mg} / \mathrm{kg}$ ). The procedures were carried out based on the guidelines of the Ethics Committee of the International Association for the Study of pain [23]. The University Research Council approved all experiments.
Following surgical preparation in the sham-operation group (Sham) the left sciatic nerve was exposed through a gluteal muscle incision and after careful homeostasis the muscle was sutured with resorbable 4/0 sutures, and the skin with $3 / 0$ nylon. In the SIL group the left sciatic nerve was exposed through a gluteal muscle incision and transected proximal to the tibio-peroneal bifurcation where a $7 \mathrm{~mm}$ segment was excised, leaving a gap about $10 \mathrm{~mm}$ due to retraction of nerve ends. Proximal and distal stumps were each inserted $2 \mathrm{~mm}$ into a silicone tube, $2 \mathrm{~mm}$ in inner diameter and $6 \mathrm{~mm}$ in outer diameter, and two 10/0 nylon sutures were placed at each end of the cuff to fix the tube in place and to leave a $10-\mathrm{mm}$ gap between the stumps. The conduit was filled with $10 \mu \mathrm{L}$ the phosphet buffered saline and sterile Vaseline was used to seal the ends of the tubes to avoid leakage. In the SIL/CsA group the conduit was filled group the conduit was filled with $10 \mu \mathrm{L}$ the carrier-drug dilution. The animals were anesthetized (see above) and euthanized with transcardial perfusion of a fixative containing $2 \%$ paraformaldehyde and $1 \%$ glutaraldehyde buffer $(\mathrm{pH}$ 7.4) 4, 8, 12 and 16 weeks after surgery.

\section{Behavioral testing}

Functional recovery of the nerve was assessed using the Basso, Beattie, and Bresnahan (BBB) locomotor rating scale for rat hind limb motor function [24]. Although BBB is widely used to assess functional recovery in spinal cord injured animals, however, it has been demonstrated that it could be most useful in assessment of never repair processes in peripheral nerve injuries [25]. Scores of 0 and 21 were given when there were no spontaneous movement and normal movement, respectively. A score of 14 shows full weight support and complete limbs coordination. BBB recordings were performed by a trained observer who was blinded to the experimental design. The testing was performed in a serene environment. The animals were observed and assessed within a course of a 4-minute exposure to an open area of a mental circular enclosure. BBB scores were recorded once before surgery in order to establish a baseline control and again weekly thereafter to assess functional recovery during 16 weeks.

\section{Functional Assessment of Reinnervation}

Sciatic functional index (SFI): Walking track analysis was performed 4, 8, 12 and 16 weeks after surgery based on Bain et al. [26]. The lengths of the third toe to its heel (PL), the first to the fifth toe (TS), and the second toe to the fourth toe (IT) were measured on the experimental side (E) and the contralateral normal side (N) in each rat. The Sciatic Function Index (SFI) in each animal was calculated by the following formula:

$\mathrm{SFI}=-38.3 \times($ EPL-NPL $) / \mathrm{NPL}+109.5 \times(\mathrm{ETS}-\mathrm{NTS}) / \mathrm{NTS}+13.3$ $\times($ EIT-NIT $) /$ NIT -8.8

In general, the SFI oscillates around 0 for normal nerve function, whereas around -100 SFI represents total dysfunction. The SFI was assessed based on the SIL group and the normal level was considered as 0 .The SFI was a negative value and a higher SFI meant the better function of the sciatic nerve.

Static sciatic index (SSI): SSI is a time-saving digitized static footprint analysis described by others [27]. A good correlation between the traditional SFI and the newly developed static sciatic 
index (SSI) and static toe spread factor (TSF), respectively, has been reported by others [27]. The SSI is a time-saving and easy technique for accurate functional assessment of peripheral nerve regeneration in rats and is calculated using the static factors, not considering the print length factor (PL), according to the equation:

$$
\mathrm{SSI}=[(108.44 \times \mathrm{TSF})+(31.85 \times \mathrm{ITSF})]-5.49
$$

Where:

$$
\text { TSF }=(\text { ETS-NTS }) / \text { NTS }
$$

$$
\mathrm{ITSF}=(\mathrm{EIT}-\mathrm{NIT}) / \mathrm{NIT}
$$

Like SFI, an index score of 0 was considered normal and an index of -100 indicated total impairment.

Electrophysiological measurement: After 16 weeks, following the track test, all animals were subjected to electrophysiological studies using Nacro bio system 320-3760 A trace 80 (USA). Under general anesthesia the left sciatic nerve was re-exposed by incision of the skin at the previous surgical site. Single electrical pulses (at supra maximal intensity) were delivered via bipolar electrodes placed in turn at the proximal and distal trunk of the regenerated nerve and electromyography (EMG) was recorded by inserting an electrode into the belly of gastrocnemius muscle. The latency and the amplitude of EMG were obtained. Also, the difference in latency of EMG was measured, and the distance between the proximal and distal sites of stimulation was measured to calculate the conduction velocity across the regenerated nerve. On the contralateral, right intact side of each animal, similar measurements were made for the determination of conduction velocity. The conduction velocity of the bridged nerve was expressed as a percentage of that on the intact side of each animal to cancel off variations between animals (\% CVR).

The recovery index of EMG amplitude in all groups was calculated based on Suzuki et al using the following formula:

Recovery index $=$ Peak amplitude of the operated side/Peak amplitude of the intact side [28].

Muscle mass: Recovery assessment was also indexed using the weight ratio of the gastrocnemius muscles 16 weeks after surgery. Immediately after sacrificing of animals, gastrocnemius muscles were dissected and harvested carefully from intact and injured sides and weighed while still wet, using an electronic balance. All measurements were made by two independent observers unaware of the analyzed group.

Histological preparation and quantitative morphometric studies: Operated nerve was dissected from surrounding tissues and a segment including several millimeters proximal and distal to the graft was harvested. Graft middle cable of Sham, TC, SIL and SIL/CsA groups were fixed in 2.5 percent glutaraldehyde. The grafts were postfixed in $\mathrm{OsO} 4(2 \%, 2 \mathrm{~h})$, dehydrated through an ethanol series and embedded in Epon. Samples were cut in $5 \mu \mathrm{m}$, stained with toluidine blue and examined under light microscopy. Morphometrical analysis was carried out using an image analyzing software (Image-Pro Express, version 6.0.0.319, Media Cybernetics, Silver Springs, MD, USA). Equal opportunity, systematic random sampling and two-dimensional dissector rules were followed in order to cope with sampling-related, fiber-location-related and fiber-size related biases [29].

\section{Statistical analysis}

Experimental results were expressed as means \pm SD. Statistical analyses were performed using PASW 18.0 (SPSS Inc., Chicago, IL, USA). Model assumptions were evaluated by examining the residual plot. Results were analyzed using a factorial ANOVA with two between-subjects factors. Bonferroni test for pairwise comparisons was used to examine the effect of time and treatments. The differences were considered significant when $\mathrm{P}<0.05$.

\section{Results}

\section{BBB recovery}

In order to assess hind limb recovery the open field locomotor was used. Figure 1 shows BBB scores compared to the baseline. All experimental groups, except for sham, showed the greatest degree of functional deficit one week after surgery. The SIL/CsA group showed significant improvement in locomotion of the operated limb compared to the control group during the study period $(\mathrm{P}<0.05)$.

\section{Recovery of sciatic nerve function and reinnervation}

SFI outcome: Figure 2 shows sciatic function index (SFI) values in experimental groups. Prior to surgery, SFI values in all groups were near zero. After the nerve transection, the mean SFI decreased to -100 due to the complete loss of sciatic nerve function in all animals. Four weeks after surgery had performed mean SFI was $-64.3 \pm-2.45$ in SIL/ CsA group, compared to $-93.65 \pm-3.50$ in SIL group. Eight weeks after surgery had carried out the improvement in SFI was observed in animals of SIL/CsA group (-59.4 \pm-3.54$)$ that was significantly higher than SIL $(-78.2 \pm-4.28)$ animals $(\mathrm{P}<0.05)$. After 12 weeks, animals of group SIL/CsA achieved a mean value for SFI of $-41.5 \pm-3.60$, whereas in group SIL, a mean value of $-60.1 \pm-3.15$ was found. At the end of the study period, 16 weeks after surgery, animals of group SIL/CsA achieved a mean value of $-33.5 \pm-3.68$ for SFI of, whereas in group SIL, a mean value of $-51.4 \pm-4.45$ was found. The statistical analyses revealed that the recovery of nerve function was significantly faster in SIL/CsA than SIL group $(\mathrm{P}<0.05)$ and, locally administered CsA promoted functional recovery.

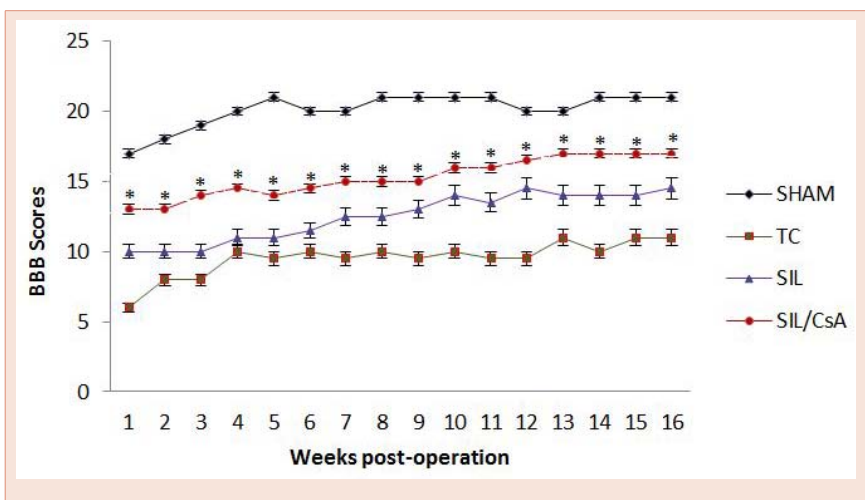

Figure 1: BBB score for all experimental groups. Local administration of CsA with silicone grafting gave better scores than in SIL group. Standard error at each data point is shown with bars. 
SSI outcome: Changes in SSI were similar to those observed in SFI, indicating significant deficit following the sciatic nerve transection (Figure 3). Changes in SSI were significant at weeks 4 , 8,12 and 16 weeks of recovery $(\mathrm{P}<0.05)$. The contrasts indicated SSI values at week 16 to differ significantly from those obtained from control, a trend also noticed for SFI $(\mathrm{P}<0.05)$.

Electrophysiology: Figures 4 and 5 show nerve conduction velocity (NCV) along regenerated sciatic nerves in experimental groups. NCV in CsA treated animals was significantly higher than that in SIL group $(\mathrm{P}<0.05)$.

Muscle mass measurement: The mean ratios of gastrocnemius muscles weight were measured. There was statistically significant difference between the muscle weight ratios of SIL/CsA and SIL groups $(\mathrm{P}<0.05)$. The results showed that in SIL/CsA group muscle weight ratio was bigger than SIL group and weight loss of the gastrocnemius muscle was ameliorated by local administration of CsA (Figure 6).

Morphological findings: Table 1 shows quantitative morphometric analyses of regenerated nerves for each of the experimental groups. 4 weeks after surgery, SIL/CsA group presented significantly greater nerve fiber, axon diameter and myelin sheath thickness compared to SIL animals $(\mathrm{P}<0.05)$. Although SIL presented regeneration patterns, the morphometric indices in SIL/CsA group both after 8,12 and 16 weeks were better than SIL (Figure 7).

Using Factorial ANOVA analysis with two between-subjects factors (Group $\times$ time); in the SIL/CsA group number of nerve fibers and myelin thickness did not show significant difference between 8,12 and 16 weeks $(P>0.05)$. Increase in mean thickness of myelin sheath did not show statistical difference between 8, 12 and 16 weeks inside each group $(\mathrm{P}>0.05)$. Mean thickness of myelin sheath from week 8 onward did not show significant difference between SIL/CsA and Sham group $(\mathrm{P}>0.05)$.

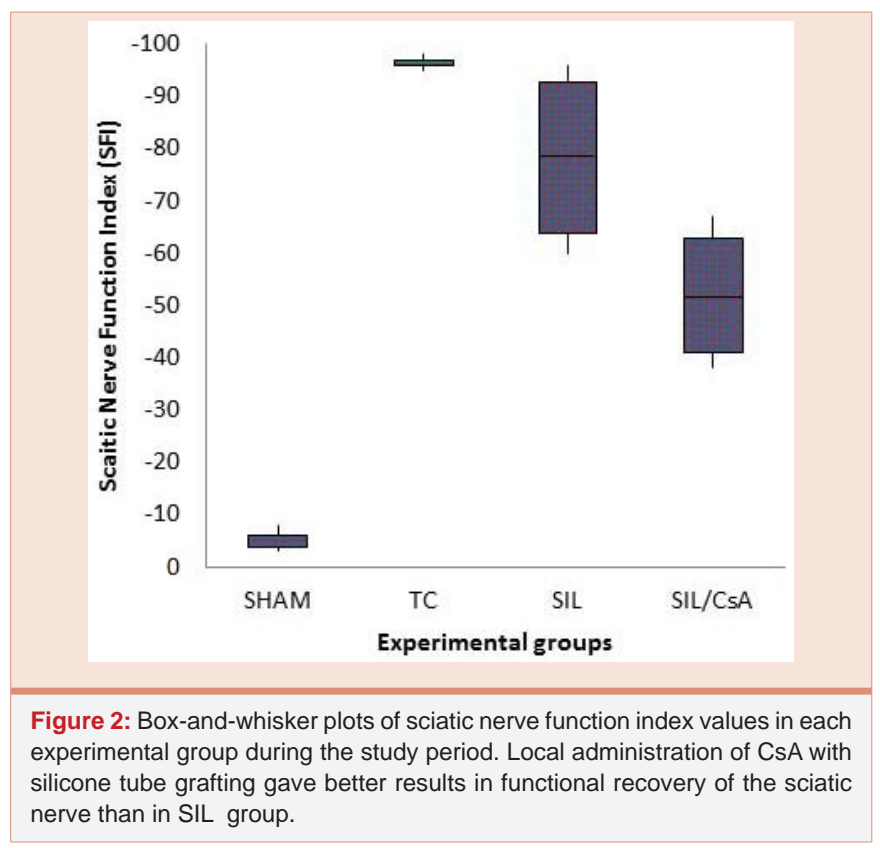

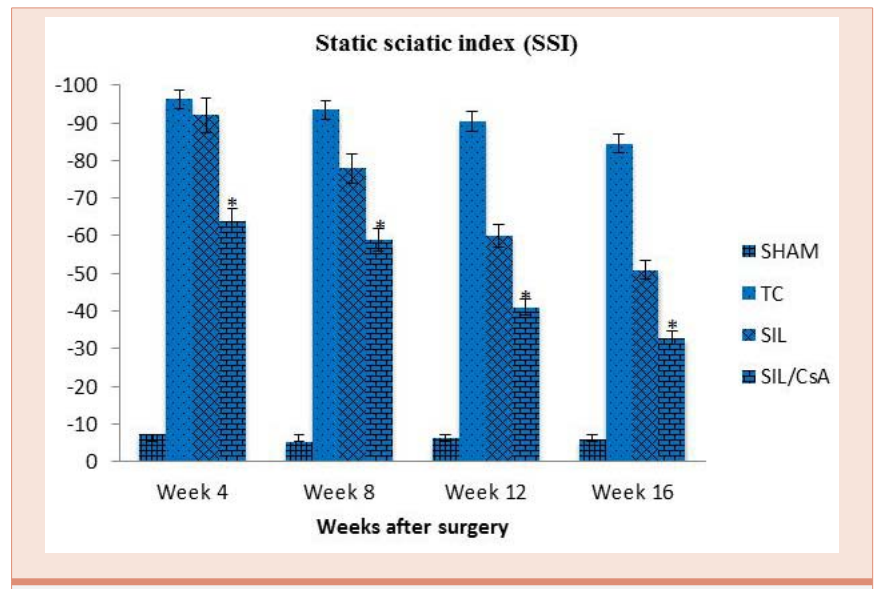

Figure 3: Bar graph indicating static sciatic index (SSI) values in each experimental group during the study period. Local administration of CsA with silicone grafting gave better results in functional recovery of the sciatic nerve than in SIL group. Data are presented as mean \pm SD. * $P<0.05$ vs SIL group.

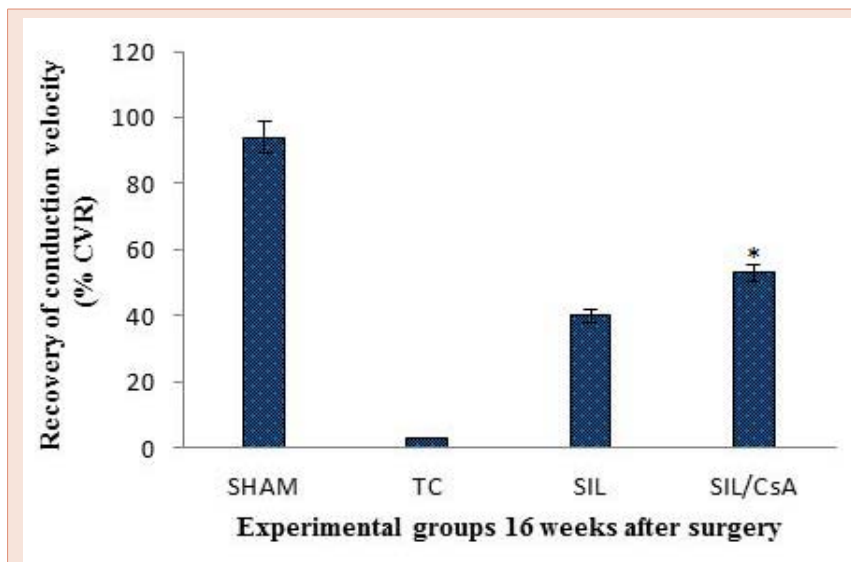

Figure 4: Percentage recovery of conduction velocity in experimental groups Data are presented as mean \pm SE. * $\mathrm{P}<0.05$ vs SIL group.

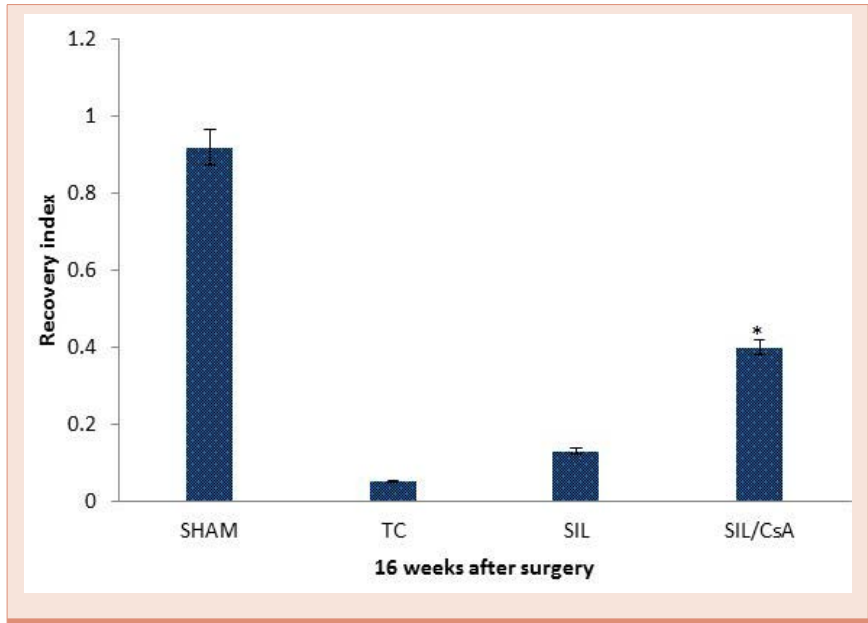

Figure 5: Recovery index in experimental groups. Data are presented as mean \pm SE. * $P<0.05$ vs SIL group. 


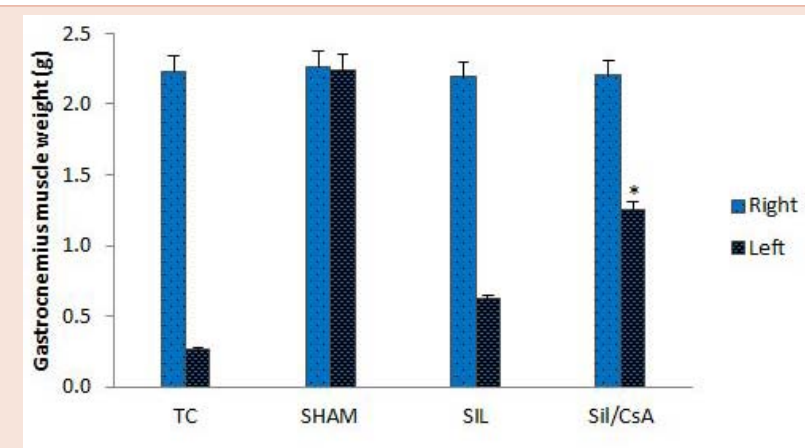

Experimental groups

Figure 6: Gastrocnemius muscle weight measurement. The gastrocnemius muscles of both sides (operated left and unoperated right) were excised and weighed in the experimental groups at 16 weeks after surgery. Data are presented as mean \pm SE. ${ }^{*} P<0.05$ vs SIL group.

Table 1: Morphometric analyses of regenerated nerves for each of the experimental groups: values are given as mean \pm SD.

\begin{tabular}{|c|c|c|c|c|c|c|c|c|}
\hline \multirow{2}{*}{ Groups } & \multicolumn{4}{|c|}{ Number of fibers } & \multicolumn{4}{|c|}{ Diameters of fibers $(\mu \mathrm{m})$} \\
\hline & 4 Weeks & 8 Weeks & 12 Weeks & 16 Weeks & 4 Weeks & 8 Weeks & 12 Weeks & 16 Weeks \\
\hline Sham & $8255 \pm 377$ & $8378 \pm 345$ & $8490 \pm 350$ & $8355 \pm 334$ & $12.07 \pm 0.17$ & $11.90 \pm 0.12$ & $12.00 \pm 0.02$ & $12.07 \pm 0.04$ \\
\hline TC & 0 & $1178 \pm 265$ & $1233 \pm 332$ & $3800 \pm 221$ & 0 & $3.90 \pm 0.50$ & $4.10 \pm 0.20$ & $6.10 \pm 0.23$ \\
\hline SIL & $1578 \pm 277$ & $3277 \pm 234$ & $3806 \pm 264$ & $3890 \pm 258$ & $3.67 \pm 0.81$ & $8.07 \pm 0.4$ & $8.53 \pm 0.72$ & $8.94 \pm 0.82$ \\
\hline SIL/CsA & $2758 \pm 229 \dagger$ & $3675 \pm 222 \dagger$ & $5471 \pm 274 †$ & $5642 \pm 250 \dagger$ & $7.43 \pm 0.40 \dagger$ & $8.67 \pm 0.59 \dagger$ & $9.88 \pm 0.85 \dagger$ & $9.84 \pm 0.68 \dagger$ \\
\hline \multirow{2}{*}{ Groups } & \multicolumn{4}{|c|}{ Diameter of axon $(\mu \mathrm{m})$} & \multicolumn{4}{|c|}{ Thickness of myelin sheath $(\mu \mathrm{m})$} \\
\hline & 4 Weeks & 8 Weeks & 12 Weeks & 16 Weeks & 4 Weeks & 8 Weeks & 12 Weeks & 16 Weeks \\
\hline Sham & $7.00 \pm 0.30$ & $6.70 \pm 0.30$ & $7.04 \pm 0.03$ & $7.05 \pm 0.15$ & $2.52 \pm 0.02$ & $2.40 \pm 0.02$ & $2.56 \pm 0.05$ & $2.55 \pm 0.05$ \\
\hline TC & 0 & $2.36 \pm 0.32$ & $2.40 \pm 0.57$ & $3.60 \pm 0.45$ & 0 & $0.82 \pm 0.14$ & $0.82 \pm 0.03$ & $1.20 \pm 0.04$ \\
\hline SIL & $2.60 \pm 0.55$ & $4.28 \pm 0.46$ & $4.88 \pm 0.36$ & $4.83 \pm 0.55$ & $0.49 \pm 0.04$ & $2.00 \pm 0.37$ & $2.16 \pm 0.25$ & $2.35 \pm 0.28$ \\
\hline SIL/CsA & $4.60 \pm 0.74 \dagger$ & $5.00 \pm 0.57 \dagger$ & $6.24 \pm 0.42 \dagger$ & $7.57 \pm 0.38 \dagger$ & $1.87 \pm 0.05 \dagger \dagger$ & $2.19 \pm 0.24$ & $2.34 \pm 0.55$ & $2.54 \pm 0.52$ \\
\hline
\end{tabular}

$\mathrm{N}$ : Number of fibers D: Diameter of fibers $(\mu \mathrm{m}) \mathrm{d}$ : Diameter of axon $(\mu \mathrm{m}) \mathrm{T}$ : Thickness of myelin sheath $(\mu \mathrm{m})$ †Results were significantly different from CHIT group $(\mathrm{P}<0.05)$
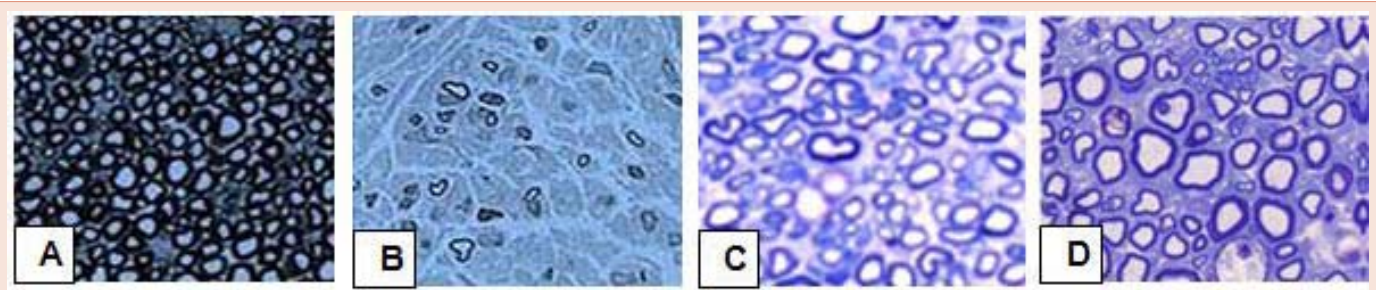

Figure 7: Light micrograph of representative cross section taken from (A) midpoint of normal sciatic nerve (SHAM), (B) regenerated cable (TC), (C) regenerated nerve (SIL) and (D) regenerated nerve (SIL/CSA) 12 weeks after surgery. (Toluidine blue, $\times 400$ ).

\section{Discussion}

Entubulation neurorrhaphy is an excellent alternative to short interposition of nerve grafts [30]. In the present study we used silicone conduit as a conduit for keeping the delivered drug in situ. It has been demonstrated that chronic administration of CsA had adverse effects on nerve regeneration [18]. However, the use of cyclosporine at early stages, 6 weeks postoperatively, has accelerated neural regeneration process [1].
Selection of an appropriate method to evaluate functional recovery of nerve regeneration is extremely influential. Walking is a coordinated activity involving sensory input, motor response and cortical integration [31]. Therefore, walking track analysis (sciatic function index) is a comprehensive test. The results of the present study showed that CsA when loaded in a silicon conduit ended up a faster and significant improvement of functional recovery of the sciatic nerve. 
Castaneda et al. [31] suggested that arrival of sprouts from the proximal stump at the distal nerve stump does not necessarily imply recovery of nerve function. Walking track analysis has frequently been used to reliably determine functional recovery following nerve repair in rat models [32,33]. Our results showed that moephometric indices were not significantly different between SIL/CSA and SIL groups after 8 weeks .In contrary, functional recovery occurred from week 8 to week 16 in SIL/CSA supporting again this idea that selection of an appropriate method to evaluate functional recovery is crucial. This study also supports the idea that the walking track analysis (SFI) is more comprehensive and reliable than histomorphometric methods in peripheral nerve repair studies [31,34].

Nerve conduction measurement is a direct evidence for the study of nerve transmission [35]. The conduction velocity depends on the diameter of axons and the thickness of myelin sheath [36]. The results of the present study showed significantly different conduction velocity between the CsA treated animals and SIL bridged regenerated sciatic nerves, therefore, the SIL conduit in combination with CsA could be assumed as a safe nerve guide with no nerve conduction interference.

As the posterior tibial branch of the sciatic nerve regenerates into the gastrocnemius muscle, it will regain its mass proportional to the amount of axonal reinnervation [37,38]. In the present study 16 weeks after surgery the muscle mass was found in both experimental groups. However, SIL/CsA group showed significantly greater ratio of the mean gastrocnemius muscle weight than SIL group indicating indirect evidence of successful end organ reinnervation.

In the histological studies, quantitative morphometrical indices of regenerated nerve fibers showed significant difference between SIL/CsA and SIL groups indicating beneficial effect of topical CsA on the nerve regeneration.

Despite much effort to introduce ideal therapeutic drugs for diabetic neuropathy, aldose reductase inhibitors, have been shown to be the most established compounds among potent drugs. However, although experimental data on aldose reductase inhibitor shave been very promising, their clinical efficacy seems limited even for mild degrees of diabetic neuropathy. One of the main problems in the usage of immunosuppressive agents is the side effects. It has been shown that CsA is able to generate reactive oxygen species and lipid peroxidation, which are directly involved in the CsA nephrotoxicity, hepatotoxicity and cardiotoxicity. Cyclosporine is also known to cause a combination of metabolic side effects including hypertension, hyperkalemia, hypercalciuria and hypomagnesemia. To overcome the drawback it has been reported to minimize the dosage or to limit the duration of therapy $[39,40]$. The ability of CsA to increase nerve regeneration in vivo is reported to be dose-related the rat in systemic administration [18]. Thus, we decided to test if topical administration of CsA would result in comparable improvement in regeneration of sciatic nerve transection models through a tubulized 10-mm gap. Results of the ability of CsA in peripheral nerve regeneration are different and sometime conflicting in the literature $[1,18,41,42]$. One possible explanation for the relative variability of the results of studies of experimental nerve injuries is the variety of models and testing methods used. Others showed that the use of cyclosporine was associated with a significant increase of fiber density and percentage of neural tissue on the distal segment of reconstructed nerves in immunosuppressed animals $[1,43]$. It has been reported that low dose administration of CsA, $5 \mathrm{mg} / \mathrm{kg}$ per day subcutaneously, increased quantitative values of fiber density [1]. Furthermore, additional action to hasten Wallerian degeneration should be taken into consideration. The Schwann cell and its basal lamina are vital components in the environment in which regenerating axons extend to grasp their peripheral targets. Schwann cells from distal stump of transected nerve start proliferating and help inflammatory infiltrating cells to eliminate debris and upregulate synthesis of trophic and tropic factors throughout wallerian degeneration, and regeneration is failed or delayed in situations where process of wallerian degeneration is diminished [38]. Host immunosuppression using cyclosporine and predegeneration of nerve grafts were useful techniques for successful installment and regeneration across nerve allografts in rabbits [41]. CsA accelerates axonal regrowth with systemic administration. However, a topical formulation that provides the same neurotrophic stimulus would be ideal because of less systemic toxicity. Entubulation neurorrhaphy using CsA loaded silicon conduit as an in situ delivery system of CsA in bridging the defects could be considered as an excellent alternative to short interposition nerve grafts. However, with the use of an interposition graft the regenerating fibers are forced to grow through scar tissue, which often results in decreased efficacy of repair and aberrant reinnervation [44]. We have not given the histological and molecular evidence for neuroprotective actionof CsA. This may be considered as a limitation of our study. Therefore, the authors stress that the current investigation to evaluate a single local dose and clinical treatment potential of CsA on nerve repair and precise mechanism of neuroprotective action of CsA in transection models remains to be investigated.

\section{Conclusion}

In conclusion, in the present study CsA was applied locally at the time of sciatic nerve repair using a silicon conduit neurorrhaphy. It demonstrated promising results in nerve regeneration in diabetic rats. Thus, dose-response studies should be conducted for Cs A to determine the combination of graft and the compound that achieve maximal efficacy in nerve transection models.

\section{Acknowledgments}

The authors would like to thank Dr. Keyvan Amini for his technical expertise.

\section{References}

1. Tuma JP, Ferreira MC, Nakamoto HA, Milcheski DA, Cheroto FA (2008) Influence of immunosupression on nerve regeneration using allografts: an experimental study on rats. Acta Ortop Bras 16: 41-44.

2. Pfister LA, Papaloizos M, Merkle HP, Gander B (2007) Nerve conduits and growth factor delivery in peripheral nerve repair. J Peripher Nerv Syst 12: 65-82.

3. Schmidt CE, Leach JB (2003) Neural tissue engineering: strategies for repair 
and regeneration. Annu Rev Biomed Eng 5: 293-347.

4. Mohammadi R, Azizi S, Delirezh N, Hobbenaghi R, Amini K (2012) Transplantation of uncultured omental adipose-derived stromal vascular fraction improves sciatic nerve regeneration and functional recovery through inside-out vein graft in rats. J Trauma 72: 390-396.

5. Battiston B, Geuna S, Ferrero M, Tos P (2005) Nerve repair by means of tubulization: literature review and personal clinical experience comparing biological and synthetic conduits for sensory nerve repair. Microsurgery 25: 258-267

6. Evans GR (2001) Peripheral nerve injury: a review and approach to tissue engineered constructs. Anat Rec 263: 396-404.

7. Dvali L, Mackinnon S-E (2003) Nerve repair, grafting, and nerve transfers. Clin Plast Surg 30: 203-221.

8. Mohammadi R, Azizi S, Delirezh N, Hobbenaghi R, Amini K (2011) Comparison of beneficial effects of undifferentiated cultured bone marrow stromal cells and omental adipose-derived nucleated cell fractions on sciatic nerve regeneration. Muscle Nerve 43: 157-163.

9. Cnpolat L, Kukner A, Canpolat I, Ozan E (1999) Ultra structural and morphometric analysis of peripheral nerve regeneration within silicone tubes. Tr J of Medical Sciences 29: 203-210.

10. Chen YS, Hsieh CL, Tsai CC, Chen TH, Cheng WC, et al. (2000) Periphera nerve regeneration using rubber chambers filled with collagen, laminin and fibronectin. Biomaterials 21: 1541-1547.

11. Lundborg G, Rosén B, Abrahamson SO, Dahlin L, Danielsen N (1994) Tubular repair of the median nerve in the human forearm. Preliminary findings. $J$ hand Surg Br 198: 273-276.

12. Chen B, Song Y-X, Liu Z-J (2009) Promotion of nerve regeneration in peripheral nerve by short-course FK506 after end-to-side neurorrhaphy. J Surg Res 152: 303-310.

13. Navarro X, Udina E, Ceballos D, Gold BG (2001) Effects of FK506 on nerve regeneration and reinnervation after graft or tube repair of long nerve gaps. Muscle Nerve 24: 905-915

14. Udina E, Rodríguez FJ, Verdú E, Espejo M, Gold BG, et al. ( 2004) FK506 enhances regeneration of axons across long peripheral nerve gaps repaired with collagen guides seeded with allogeneic Schwann cells. Glia 47: 120129.

15. Azizi S, Mohammadi R, Amini K, Fallah R (2012) Effects of topically administered FK506 on sciatic nerve regeneration and reinnervation after vein graft repair of short nerve gaps. Neurosurg Focus 32: E5

16. White D-J-G, Calne R-Y (1982) The use of cyclosporine A immunosuppression in organ grafting. Immunological Rev 65: 115-131.

17. Bain JR, Mackinnon SE, Hudson AR, Falk RE, Falk JA, et al. (1988) The peripheral nerve allograft: $A$ dose- response curve in the rat immunosuppressed with cyclosporine A. Plast Reconstr Surg 82: 447-457.

18. Meirer R, Babuccu O, Unsal M, Nair DR, Gurunluoglu R, et al. (2002) Effect of chronic cyclosporine administration on peripheral nerve regeneration: a dose-response study. Ann Plast Surg 49: 96-103.

19. Taskinen H-S, Roytta M (2000) Cyclosporin A affects axons and macrophages during Wallerian degeneration. J Neurotrauma 17: 431-440.

20. Wang MS, Zeleny - Pooley M, Gold BG (1997) Comparative dose-dependence study of FK506 and cyclosporin A on the rate of axonal regeneration in the rat sciatic nerve. J Pharmacol Exp Ther 282: 1084-1093.

21. Bain JR, Mackinnon SE, Hudson AR, Falk RE, Falk JA, et al. (1989) Preliminary report of peripheral nerve allografting in primates immunosuppressed with cyclosporin A.Transplant Proc 21: 3176-3177.

22. Bain JR, Mackinnon SE, Hudson AR, Falk RE, Falk JÁ, et al. (1988) The peripheral nerve allograft: an assessment of regeneration across nerve allografts in rats immunosuppressed with cyclosporin A. Plast Reconstr Surg 82: 1052-1066.

23. Zimmermann M (1983) Ethical guidelines for investigations of experimental pain in conscious animals. Pain 16: 109-110

24. Basso DM, Beattie MS, Bresnahan JC (1995) A sensitive and reliable locomotor rating scale for open field testing in rats. J Neurotrauma 12: 1-21.

25. Dinh P, Hazel A, Palispis W, Suryadevara S, Gupta R (2009) Functional assessment after sciatic nerve injury in a rat model. Microsurgery 29: 644 $-649$

26. Bain JR, Mackinnon SE, Hunter DA (1989) Functional evaluation of complete sciatic, peroneal, and posterior tibial nerve lesions in the rat. Plast Reconstr Surg 83: 129-138.

27. Bervar M (2000) Video analysis of standing-an alternative footprint analysis to assess functional loss following injury to the rat sciatic nerve. J Neurosci Meth 102: 109-16.

28. Di Benedetto G, Zura G, Mazzucchelli R, Santinelli A, Scarpelli M, et al. (1998) Nerve regeneration through a combined autologous conduit (vein plus acellular muscle grafts). Biomaterials 19: 173-181.

29. Geuna S, Gigo-Benato D, Rodrigues AC (2004) On sampling and sampling errors in histomorphometry of peripheral nerve fibers. Microsurgery 24: 72 76.

30. Diaz LM, Steele MH, Guerra AB, Aubert FE, Sloop GD, et al. ( 2004) The role of topically administered FK506 (tacrolimus) at the time of facial nerve repair using entubulation neurorrhaphy in a rabbit model. Ann Plast Surg 2: 407 413.

31. Castañeda F, Kinne RK (2002) Omental graft improves functional recovery of transected peripheral nerve. Muscle Nerve 26: 527-532.

32. Bain JR, Mackinnon SE, Hunter DA (1989) Functional evaluation of complete sciatic, peroneal, and posterior tibial nerve lesions in the rat. Plast Reconstr Surg 83: 129-138.

33. De Medinaceli L, Freed WJ, Wyatt RJ (1982) An index of the functional condition of rat sciatic nerve based on measurements made from walking tracks. Exp Neurol 77: 634-643.

34. Munro CA, Szalai JP, Mackinnon SE, Midha R (1998) Lack of association between outcome measures of nerve regeneration. Muscle Nerve 21: 10951097.

35. Matsumoto K, Ohnishi K, Kiyotani T, Sekine T, Ueda H, et al. (2000) Peripheral nerve regeneration across an $80-\mathrm{mm}$ gap bridged by a polyglycolic acid (PGA)-collagen tube filled with laminin-coated collagen fibers: a histological and electrophysiological evaluation of regenerated nerves. Brain Res 868: 315-328.

36. Chen CJ, Ou YC, Liao SL, Chen WY, Chen SY, et al. (2007) Transplantation of bone marrow stromal cells for peripheral nerve repair. Exper Neurol 204: 443-453.

37. Evans GR, Brandt K, Widmer MS, Lu L, Meszlenyi RK, et al. (1999) In vivo evaluation of poly(L-lactic acid) porous conduits for peripheral nerve regeneration. Biomaterials 20: 1109-1115.

38. Hou Z, Zhu J (1998) An experimental study about the incorrect electrophysiological evaluation following peripheral nerve injury and repair. Electromyogr Clin Neurophysiol 38: 301-304.

39. Melnikov S, Mayan H, Uchida S, Holtzman EJ, Farfel Z (2011) Cyclosporine metabolic side effects: association with the WNK4 system. Eur J Clin Invest 41: 1113-1120.

40. Rezzani R (2006) Exploring cyclosporine A-side effects and the protective 
role-played by antioxidants: the morphological and immunohistochemical studies. Histol Histopathol 21: 301-316.

41. de la Monte SM, Bour C, Radhakrishnan VV, Jupiter JB, Smith RJ, et al (1988) Effects of cyclosporine $A$ and predegeneration on survival and regeneration of peripheral nerve allografts in rabbits. Surg Neurol 29: 95-100.

42. Muramatsu K, Doi K, Kawai S (1995) Nerve regenerating effect of short-course administration of cyclosporine after fresh peripheral nerve allotransplantation in the rat: comparison of nerve regeneration using different forms of donor nerve allografts. Microsurgery 16: 496-504.

43. Berger A, Lassner F, Schaller E (1996) Experimental nerve allografting World plast 1: 330-339.

44. Weber RA, Breidenbach WC, Brown RE, Jabaley ME, Mass DP (2000) A randomized prospective study of polyglycolic acid conduits for digital nerve reconstruction in humans. Plast Reconstr Surg 106: 1036-1045. 